\title{
A multiple scattering interaction analysis of intersecting waveguides
}

\author{
Niraj Agrawal, L. McCaughan, ${ }^{\text {a) }}$ and S. R. Seshadri \\ Department of Electrical and Computer Engineering, University of Wisconsin, Madison, Wisconsin 53706
}

(Received 16 March 1987; accepted for publication 26 May 1987)

\begin{abstract}
A multiple scattering technique is developed for describing the physics of intersecting waveguides. In this picture the two waveguides interact by scattering the fields of each other in a self-consistent manner. The development of such an analysis is motivated by the fact that the previous theory, which is based on the idea of symmetric and antisymmetric modes of the composite waveguide structure, has a limited validity. The analysis reveals new features of this geometry with important device applications in integrated optics.
\end{abstract}

\section{INTRODUCTION}

An intersecting waveguide structure consists of two straight waveguides crossing each other at a small angle. This coupled waveguide geometry has recently been added to the list of basic elements used for integrated optics. ${ }^{1-4}$ Electro-optical switches have been demonstrated in lithium niobate with the addition of electrodes to intersecting waveguides. ${ }^{3}$ A nonblocking $4 \times 4$ switch array with bow-tie-type geometry has also been fabricated with this waveguide structure. ${ }^{4}$ The simplicity of geometry, i.e., absence of $S$ bends, makes it possible to achieve higher levels of integration with a switch based on this geometry than is possible with directional couplers. ${ }^{5}$ Intersecting waveguides also offer the possibility of producing novel switch array interconnections, such as the Benes or Clos networks. ${ }^{5}$

The previous theory for single-mode intersecting waveguides invokes the concept of interference between the lowest-order symmetric and antisymmetric modes of this composite waveguide structure. ${ }^{3,6}$ These local modes are usually constructed by a superposition of the fundamental mode of the individual waveguides. Such an analysis has at best a limited validity for intersecting waveguides because the optical fields of the two modes which are superposed do not propagate in the same direction. In this paper we present a systematic theory which describes for the first time the physics of two intersecting waveguides correctly. The theory of coupling between two intersecting waveguides is formulated in terms of a multiple scattering interaction picture and is discussed in Sec. II. In this picture the two waveguides interact by scattering the fields of each other in a self-consistent manner. The applicability of the proposed theory is quite general in its scope and is not limited to the case of two intersecting waveguides. In Sec. III the directional coupler is treated to provide a clear understanding of the idea behind the multiple scattering interaction analysis. In Sec. IV the coupling between two identical intersecting waveguides is presented along with a description of the distinguishing features of the solution obtained. The theory described in this paper can also be used to describe a variety of other phenomenon in intersecting waveguides. For example, the analysis of an entire class of novel devices which may be designed by employing a variety of index distributions in the common

a) Author to whom correspondence should be addressed. volume region. Some concluding remarks are given in Sec. V.

\section{THE MULTIPLE SCATTERING INTERACTION ANALYSIS}

This method utilizes a multiple scattering interaction picture of coupling between the two waveguides and is formulated in terms of the Green's function for the individual waveguides. The wave equation for the transverse electric (TE) mode, $\partial / \partial x=0, \nabla_{t}^{2} \equiv\left(\partial^{2} / \partial y^{2}\right)+\left(\partial^{2} / \partial z^{2}\right)$, reduces to

$$
\left[\nabla_{t}^{2}+\omega^{2} \mu \epsilon(y, z)\right] E_{x}(y, z)=0,
$$

with a harmonic time dependence $\exp (-i \omega t)$, and dielectric permittivity

$$
\epsilon(y, z)=\epsilon_{s}+\epsilon_{1}(y, z)+\epsilon_{2}(y, z)+\iota(y, z),
$$

where $\epsilon_{s}$ pertains to the substrate, $\epsilon_{1,2}(y, z)$ represent the two intersecting single-mode waveguides, and $\iota(y, z)$ is used to incorporate an arbitrary scattering inhomogeneity in the intersection region. The total field $E_{x}(y, z)$ is decomposed in terms of fields $E_{x, i}(y, z)$ associated with the two waveguides with $i=1,2$. Equation (1) is rewritten as

$$
\begin{aligned}
{\left[\nabla_{t}^{2}+\right.} & \left.\omega^{2} \mu\left(\epsilon_{s}+\epsilon_{i}\right)\right] E_{x, i} \\
= & -\omega^{2} \mu\left(\epsilon_{i} E_{x, j}+\epsilon_{j} E_{x, i}\right)-\omega^{2} \mu \iota\left(E_{x, i}+E_{x, j}\right) \\
& -\left[\nabla_{t}^{2}+\omega^{2} \mu\left(\epsilon_{s}+\epsilon_{j}\right)\right] E_{x, j},
\end{aligned}
$$

with $i=1, j=2$, and $i=2, j=1$. In what follows, the subscripts 1,2 are used to denote a point $(y, z)$ in the $\left(y_{1}, z_{1}\right)$ and $\left(y_{2}, z_{2}\right)$ coordinate axes, respectively (see Fig. 1 ). In addition, a source point is distinguished from an observation point by a prime superscript.

A wave is launched from the left of the junction at $z_{1} \rightarrow-\infty$ in waveguide 1 . The fields in the absence of coupling between the waveguides 1 and 2 are

$$
\begin{aligned}
& E_{x, 1}(y, z)=E_{x, 1}^{(0)}(y, z), \\
& E_{x, 2}(y, z)=E_{x, 2}^{(0)}(y, z)=0,
\end{aligned}
$$

where the superscript within parenthesis denotes the order of scattering interaction. However, in the presence of a coupling between the two waveguides, a part of the power is scattered from waveguide 1 into 2 , and this power can be calculated from the first-order scattered field $E_{x, 2}^{(1)}(y, z)$. Substituting Eq. (4) into Eq. (3) with $i=2, j=1$ leads to 


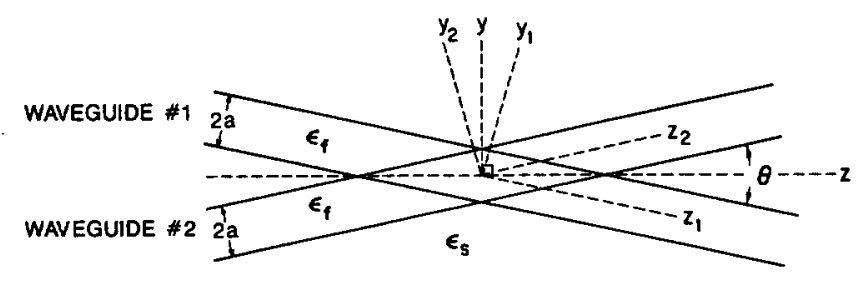

FIG. 1. Schematic diagram of the coordinate axes for the analysis of intersecting waveguides.

$$
\left[\nabla_{t}^{2}+\omega^{2} \mu\left(\epsilon_{s}+\epsilon_{2}\right)\right] E_{x, 2}^{(1)}=-\omega^{2} \mu\left(\epsilon_{2}+\iota\right) E_{x, 1}^{(0)},
$$

where the term on the right-hand side accounts for an exchange of power between the two waveguides in the first order. The fields in the two waveguides at the end of this virtual interaction are given by

$$
\begin{aligned}
& E_{x, 1}(y, z)=E_{x, 1}^{(0)}(y, z), \\
& E_{x, 2}(y, z)=E_{x, 2}^{(0)}(y, z)+E_{x, 2}^{(1)}(y, z) .
\end{aligned}
$$

In the next stage of interaction, the field $E_{x, 2}^{(1)}(y, z)$ is scattered into waveguide 1 and is defined to be $E_{x, 1}^{(1)}(y, z)$. Substituting Eq. (6) into Eq. (3), with $i=1, j=2$ leads to

$$
\left[\nabla_{t}^{2}+\omega^{2} \mu\left(\epsilon_{s}+\epsilon_{1}\right)\right] E_{x, 1}^{(1)}=-\omega^{2} \mu\left(\epsilon_{1}+\imath\right) E_{x, 2}^{(1)} \text {. }
$$

The field $E_{x, 1}^{(1)}(y, z)$ is in turn scattered into $E_{x, 2}^{(2)}(y, z)$ in waveguide 2 and so on. The wave equation satisfied by the $n$th order scattered fields $E_{x, 2}^{(n)}(y, z)$ and $E_{x, 1}^{(n)}(y, z)$ can be derived in a manner similar to Eqs. (5) and (7) by the induction method and are expressed as

$$
\begin{aligned}
& {\left[\nabla_{t}^{2}+\omega^{2} \mu\left(\epsilon_{s}+\epsilon_{2}\right)\right] E_{x, 2}^{(n)}=-\omega^{2} \mu\left(\epsilon_{2}+\imath\right) E_{x, 1}^{(n-1)},} \\
& {\left[\nabla_{t}^{2}+\omega^{2} \mu\left(\epsilon_{s}+\epsilon_{1}\right)\right] E_{x, 1}^{(n)}=-\omega^{2} \mu\left(\epsilon_{1}+\iota\right) E_{x, 2}^{(n)} .}
\end{aligned}
$$

The total field in waveguides 1,2 can be written as a sum of fields due to an infinite sequence of scattering interactions with $i=1,2$ as

$$
E_{x, i}(y, z)=\sum_{n=0}^{\infty} E_{x, i}^{(n)}(y, z)
$$

The solution to Eqs. ( $8 \mathrm{a}$ ) and ( $8 \mathrm{~b}$ ) can be expressed in terms of Green's function $G_{i}\left(y, z ; y^{\prime}, z^{\prime}\right)$ of the two slab waveguides with $i=1,2$ as

$$
\begin{aligned}
E_{x, 2}^{(n)}(y, z)= & \frac{i \omega}{4} \iint\left[\epsilon_{2}\left(y^{\prime}, z^{\prime}\right)+\iota\left(y^{\prime}, z^{\prime}\right)\right] E_{x, 1}^{(n-1)}\left(y^{\prime}, z^{\prime}\right) \\
& \times G_{2}\left(y, z ; y^{\prime}, z^{\prime}\right) d y^{\prime} d z^{\prime} \\
E_{x, 1}^{(n)}(y, z)= & \frac{i \omega}{4} \iint\left[\epsilon_{1}\left(y^{\prime}, z^{\prime}\right)+\iota\left(y^{\prime}, z^{\prime}\right)\right] E_{x, 2}^{(n)}\left(y^{\prime}, z^{\prime}\right) \\
& \times G_{1}\left(y, z ; y^{\prime}, z^{\prime}\right) d y^{\prime} d z^{\prime}
\end{aligned}
$$

where the Green's function $G_{i}\left(y, z ; y^{\prime}, z^{\prime}\right)$ with $i=1,2$ is defined by

$$
\left[\nabla_{t}^{2}+\omega^{2} \mu\left(\epsilon_{s}+\epsilon_{1}\right)\right] G_{i}\left(y, z ; y^{\prime}, z^{\prime}\right)=i 4 \omega \mu \delta\left(y-y^{\prime}, z-z^{\prime}\right) .
$$

We restrict our attention to the coupling between the $\mathrm{TE}_{0}$ (fundamental) modes of the two intersecting slab waveguides denoted by $\Psi_{i}\left(y_{i}, z_{i}\right)$ with $i=1,2$. The Green's func- tion is first obtained in its Fourier integral representation in the complex wave number plane and then the inverse Fourier transform is taken including the contribution from only a single pole corresponding to the $\mathrm{TE}_{0}$ mode of the slab waveguide. It is found that the Green's function $G_{i}\left(y_{i}, z_{i} ; y_{i}^{\prime}, z_{i}^{\prime}\right)$ for the two single-mode slab waveguides with $i=1,2$ can be expressed in terms of the eigenfunctions as

$$
G_{i}\left(y_{i}, z_{i} ; y_{i}^{\prime}, z_{i}^{\prime}\right)=\Psi_{i}^{*}\left(y_{i}^{\prime}, z_{i}^{\prime}\right) \Psi_{i}\left(y_{i}, z_{i}\right) .
$$

Each point source implied in Eqs. (8a) and (8b) excites the fundamental mode $\mathrm{TE}_{0}$ mode of the waveguides 2,1 traveling in both $\pm z_{2}, \pm z_{1}$ directions. The formalism described here is quite general and can be used to analyze directional couplers as well, which are formed with two parallel waveguides. In the two special cases of multiple scattering interaction analysis to be considered in this paper, the two waveguides that are coupled are either parallel or intersect each other at a small angle (see Secs. III and IV). In either case the contradirectional coupling in all orders of scattering interaction is small because of a large mismatch between the propagation wave vectors of the incident and reflected waves along $+\dot{z}_{1}$ and $-z_{2}$ (or $+z_{2}$ and $-z_{1}$ ) directions, respectively. Equation (10a) represents a weak coupling over an extended region of interaction in the $n$th order and causes the envelope of the eigenfunction $\Psi_{2}$ corresponding to the $\mathrm{TE}_{0}$ mode to grow slowly along the axis of waveguide 2 . To obtain the value of the envelope $A{ }_{2}^{(n)}$ at a point $\left(y_{2}, z_{2}\right)$, the $y_{2}^{\prime}$ integral extends over all space but the $z_{2}^{\prime}$ integral is evaluated from $-\infty$ to $z_{2}$. A similar remark holds for the scattering interaction represented by Eq. (10b). Since the Green's function for a single mode waveguide is separable in source and observation point coordinates, i.e., $\mathbf{r}^{\prime}$ and $\mathbf{r}$ respectively, the $n$th order scattered fields can be written in the form

$$
\begin{aligned}
& E_{x, 2}^{(n)}\left(y_{2}, z_{2}\right)=A_{2}^{(n)}\left(z_{2}\right) \Psi_{2}\left(y_{2}, z_{2}\right), \\
& E_{x, 1}^{(n)}\left(y_{1}, z_{1}\right)=A_{1}^{(n)}\left(z_{1}\right) \Psi_{1}\left(y_{1}, z_{1}\right) .
\end{aligned}
$$

In fact, Eqs. (12a) and (12b) form the basis of the physical picture of spatial evolution of fields along the axes of waveguides 1 and 2 in the intersecting waveguide structure as discussed above.

Finally, the explicit expressions for some of the quantities referred to earlier in this section are given for the specific case of step index slab waveguides. The two slab waveguides with $i=1,2$ are described as

$$
\epsilon_{i}\left(y_{i}, z_{i}\right)=\left\{\begin{array}{lc}
\epsilon_{f, i}-\epsilon_{s} & \text { if } \quad-a \leqslant y_{i} \leqslant+a, \\
0 & \text { otherwise. }
\end{array}\right.
$$

The normalized (unit power) eigenfunctions $\Psi_{i}\left(y_{i}, z_{i}\right)$ of the two waveguides corresponding to $\mathrm{TE}_{0}$ mode with $i=1,2$ are as follows:

$$
\begin{aligned}
\Psi_{i}\left(y_{i}, z_{i}\right)= & \frac{\sqrt{2 \omega \mu} e^{-\gamma_{i}\left(y_{i}-a\right)} e^{i \beta_{z_{i}}}}{\sqrt{\beta_{i}} \sqrt{\left(a+1 / \gamma_{i}\right)} \sqrt{\left(1+\gamma_{i}^{2} / \alpha_{i}^{2}\right)}} \\
& \text { for }+a \leqslant y_{i}<\infty, \\
\Psi_{i}\left(y_{i}, z_{i}\right)= & \frac{\sqrt{2 \omega \mu} \cos \left(\alpha_{i} y_{i}\right) e^{i \beta_{z_{i}}}}{\sqrt{\beta_{i}} \sqrt{\left(a+1 / \gamma_{i}\right)}} \\
& \text { for }-a \leqslant y_{i} \leqslant+a,
\end{aligned}
$$




$$
\begin{aligned}
\Psi_{i}\left(y_{i}, z_{i}\right)= & \frac{\sqrt{2 \omega \mu} e^{\gamma_{i}\left(y_{i}+a\right)} e^{i \beta_{z_{i}}}}{\sqrt{\beta_{i}} \sqrt{\left(a+1 / \gamma_{i}\right)} \sqrt{1+\gamma_{i}^{2} / \alpha_{i}^{2}}} \\
& \text { for }-\infty<y_{i} \leqslant-a .
\end{aligned}
$$

The following relations are satisfied by the constants $\alpha_{i}, \beta_{i}, \gamma_{i}$ :

$$
\begin{aligned}
& \alpha_{i}^{2}=\omega^{2} \mu \epsilon_{f, i}-\beta_{i}^{2}, \\
& \gamma_{i}^{2}=\beta_{i}^{2}-\omega^{2} \mu \epsilon_{s}, \\
& \gamma_{i}=\alpha_{i} \tan \left(\alpha_{i} a\right),
\end{aligned}
$$

where Eq. (15c) is the dispersion relation for even modes of the slab waveguides, of which the $\mathrm{TE}_{0}$ mode is a special case.

\section{ANALYSIS OF THE DIRECTIONAL COUPLER}

An analysis based on the multiple scattering interaction picture is presented in this section for coupling between parallel waveguides so as to provide an interpretation of the scattered fields in various orders of interaction and relate them to the results obtained using coupled mode theory. The equations derived in Sec. II can be readily applied, the only difference being that the three coordinate systems, instead of intersecting each other at a common point, are now parallel along the axes of the two waveguides and are displaced with respect to each other in the transverse direction (see Fig. 2). In addition, the wave is launched from the left at $z_{1}=0$ in waveguide 1 .

In case of coupling between parallel waveguides, it is not possible to specify the input field amplitude in both the waveguides independently. To be specific, the excitation conditions given by Eqs. (4a) and (4b) are strictly speaking not valid in the present case. However, as the interwaveguide separation is made larger and larger for a pair of nearly identical waveguides, the conditions given by Eqs. (4a) and (4b) provide a better and better approximation to the physical reality. We assume these conditions to be valid in this analysis. Substituting Eq. (11) in Eq. (10a) with $n=1$ and the relation

$$
E_{x, 1}^{(0)}(y, z)=\Psi_{1}(y, z)
$$

gives

$$
\begin{aligned}
E_{x, 2}^{(1)}(y, z)= & \left(\frac{i \omega}{4} \iint \epsilon_{2}\left(y^{\prime}, z^{\prime}\right) \Psi_{1}\left(y^{\prime}, z^{\prime}\right)\right. \\
& \left.\times \Psi_{2}^{*}\left(y^{\prime}, z^{\prime}\right) d y^{\prime} d z^{\prime}\right) \Psi_{2}(y, z) .
\end{aligned}
$$

Since the two waveguides are parallel in the present case, there is no intersection region. Therefore, in deriving Eq. (16b), the value of $\iota(y, z)$ is taken to be zero everywhere. The integral in this equation is evaluated by using Eqs. (13)-

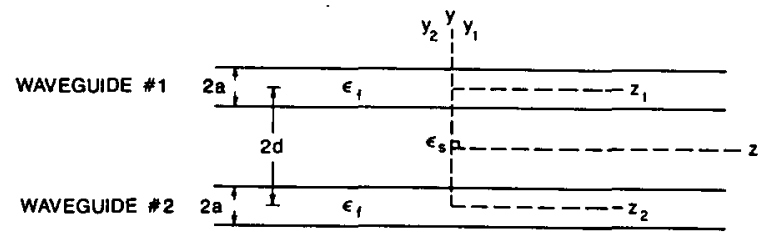

FIG. 2. Schematic diagram of the coordinate axes for the analysis of parallel waveguides.
(15) to obtain the first-order scattered field in waveguide 2 as

$$
E_{x, 2}^{(1)}(y, z)=i \kappa_{12} z \Psi_{2}(y, z),
$$

with

$$
\kappa_{12}=\frac{\gamma e^{-2 \gamma(d-a)}}{\beta(a+1 / \gamma) \sqrt{\left(1+\gamma^{2} / \alpha_{1}^{2}\right)\left(1+\gamma^{2} / \alpha_{2}^{2}\right)}} .
$$

In deriving Eq. (17), the phase-matching condition $\beta_{1}=\beta_{2} \equiv \beta$ has been assumed, which when combined with Eq. (15b) implies that $\gamma_{1}=\gamma_{2} \equiv \gamma$. The fields in both the waveguides can be obtained to all orders of scattering interaction in such a case and for $n \geqslant 1$ are given by

$$
\begin{aligned}
& E_{x, 2}^{(n)}(y, z)=\sqrt{\kappa_{12} / \kappa_{21}} \frac{\left(i \sqrt{\kappa_{12} \kappa_{21}} z\right)^{2 n-1}}{(2 n-1) !} \Psi_{2}(y, z), \\
& E_{x, 1}^{(n)}(y, z)=\frac{\left(i \sqrt{\kappa_{12} \kappa_{21}} z\right)^{2 n}}{(2 n) !} \Psi_{1}(y, z),
\end{aligned}
$$

where $\kappa_{21}$ can be obtained from $\kappa_{12}$ by interchanging the subscripts 1,2. The relation between $\kappa_{12}$ and $\kappa_{21}$ follows from Eq. (17b) to be

$$
\kappa_{12}=\kappa_{21} \equiv \kappa \text {. }
$$

Equations (4), (9), (16), (18), and (19) can be used to obtain the total field in the two waveguides as

$$
\begin{aligned}
& E_{x, 2}(y, z)=i \sin (\kappa z) \Psi_{2}(y, z), \\
& E_{x, 1}(y, z)=\cos (\kappa z) \Psi_{1}(y, z) .
\end{aligned}
$$

If the phase-matching condition is not satisfied, the coupling between the two waveguides is not symmetric under the interchange of subscripts 1,2 . Moreover, no general expressions can be written to evaluate the $n$th order scattering interaction term in this case. The sum of the first three terms in the two waveguides yields a solution which is correct up to $\sim(\kappa z)^{4}$ with $\kappa=\sqrt{\kappa_{12} \kappa_{21}}, \eta \equiv \frac{1}{2}\left(\beta_{1}-\beta_{2}\right)$ and is obtained as

$$
\begin{aligned}
E_{x, 2}(y, z)= & E_{x, 2}^{(0)}(y, z)+E_{x, 2}^{(1)}(y, z)+E_{x, 2}^{(2)}(y, z) \\
= & \sqrt{\kappa_{12} / \kappa_{21}}\left[i \kappa z-\kappa \eta z^{2}-\frac{i \kappa^{3} z^{3}}{6}-\frac{i \kappa \eta^{2} z^{3}}{3}\right. \\
& \left.+\frac{\kappa^{3} \eta z^{4}}{6}+\frac{\kappa \eta^{3} z^{4}}{3}+O\left(\kappa^{5} z^{5}\right)\right] \Psi_{2}(y, z), \\
E_{x, 1}(y, z)= & E_{x, 1}^{(0)}(y, z)+E_{x, 1}^{(1)}(y, z)+E_{x, 1}^{(2)}(y, z) \\
= & {\left[1-\frac{\kappa^{2} z^{2}}{2}+\frac{i \kappa^{2} \eta z^{3}}{3}+\frac{\kappa^{4} z^{4}}{24}+\frac{\kappa^{2} \eta^{2} z^{4}}{6}\right.} \\
& \left.+O\left(\kappa^{5} z^{5}\right)\right] \Psi_{1}(y, z) .
\end{aligned}
$$

The solution obtained using the coupled mode theory analysis of the same problem is?

$$
\begin{aligned}
E_{x, 2}(y, z)= & i \sqrt{\kappa_{12} / \kappa_{21}} \frac{\kappa}{\sqrt{\kappa^{2}+\eta^{2}}} e^{i \eta z} \\
& \times \sin \left(\sqrt{\kappa^{2}+\eta^{2}} z\right) \Psi_{2}(y, z), \\
E_{x, 1}(y, z)= & e^{-i \eta z}\left(\cos \left(\sqrt{\kappa^{2}+\eta^{2}} z\right)+\frac{i \eta}{\sqrt{\kappa^{2}+\eta^{2}}}\right. \\
& \left.\times \sin \left(\sqrt{\kappa^{2}+\eta^{2}} z\right)\right) \Psi_{1}(y, z) .
\end{aligned}
$$


Equations (22a) and (22b) can be expanded into power series in $\kappa z$. It is found that such power series representations of Eqs. (22a) and (22b) are identical to the expansions in Eqs. (21a) and (21b) up to $\sim(\kappa z)^{4}$. It can be shown by evaluating still higher-order terms given by Eqs. (10a) and (10b) that Eqs. (22a) and (22b) represent the exact sum of the series given in Eq. (9) with $i=2,1$ for the coupling between two parallel waveguides. It is evident from the analysis presented in this section that the multiple scattering interaction analysis yields results which are in agreement with the results obtained using coupled mode theory.

\section{ANALYSIS OF COUPLING BETWEEN TWO INTERSECTING WAVEGUIDES}

An analysis based on multiple scattering interaction is presented in this section for coupling between two identical waveguides intersecting at an angle $\theta$. The differential and integrated coupling coefficients are defined, respectively, as

$$
\begin{aligned}
\kappa_{i j}\left(z_{j}^{\prime}\right)= & \frac{\omega}{4} \int_{-\infty}^{\infty}\left[\epsilon_{j}\left(y_{j}^{\prime}, z_{j}^{\prime}\right)+\iota\left(y_{j}^{\prime}, z_{j}^{\prime}\right)\right] \\
& \times \Psi_{i}\left(y_{i}^{\prime}, z_{i}^{\prime}\right) \Psi_{j}^{*}\left(y_{j}^{\prime}, z_{j}^{\prime}\right) d y_{j}^{\prime}, \\
K_{i j}\left(z_{j}\right)= & \int_{-\infty}^{z_{j}} \kappa_{i j}\left(z_{j}^{\prime}\right) d z_{j}^{\prime},
\end{aligned}
$$

with $i=1, j=2$ and $i=2, j=1$. It can be shown by performing the integrals in Eqs. (23a) and (23b) that the functional dependence of $\kappa_{i j}\left(K_{i j}\right)$ on its argument $z_{j}$ is same as that of $\kappa_{j i}\left(K_{j i}\right)$ on its argument $z_{i}$. This symmetry property is symbolically expressed as

$$
\begin{aligned}
& \kappa_{i j}(.)=\kappa_{j i}(.) \equiv \kappa(.), \\
& K_{i j}(.)=K_{j i}(.) \equiv K(.) .
\end{aligned}
$$

Substituting Eq. (11) in Eq. (10a) with $n=1$ and the relation

$$
E_{x, 1}^{(0)}\left(y_{1}, z_{1}\right)=\Psi_{1}\left(y_{1}, z_{1}\right)
$$

gives

$$
\begin{aligned}
E_{x, 2}^{(1)}\left(y_{2}, z_{2}\right)= & \left(\frac{i \omega}{4} \iint\left[\epsilon_{2}\left(y_{2}^{\prime}, z_{2}^{\prime}\right)+\iota\left(y_{2}^{\prime}, z_{2}^{\prime}\right)\right]\right. \\
& \left.\times \Psi_{1}\left(y_{1}^{\prime}, z_{1}^{\prime}\right) \Psi_{2}^{*}\left(y_{2}^{\prime}, z_{2}^{\prime}\right) d y_{2}^{\prime} d z_{2}^{\prime}\right) \\
& \times \Psi_{2}\left(y_{2}, z_{2}\right) .
\end{aligned}
$$

The integral in Eq. (25b) is evaluated using Eqs. (13) and (14) and (23) and (24) to obtain the first-order scattered field in waveguide 2 in the form

$$
E_{x, 2}^{(1)}\left(y_{2}, z_{2}\right)=i K\left(z_{2}\right) \Psi_{2}\left(y_{2}, z_{2}\right)
$$

Substituting Eqs. (11) and (26) in Eq. (10b) with $n=1$ gives

$$
\begin{aligned}
E_{x, 1}^{(1)}\left(y_{1}, z_{1}\right)= & \left(\frac{i \omega}{4} \iint\left[\epsilon_{1}\left(y_{1}^{\prime}, z_{1}^{\prime}\right)+\iota\left(y_{1}^{\prime}, z_{1}^{\prime}\right)\right] i K\left(z_{2}^{\prime}\right)\right. \\
& \left.\times \Psi_{2}\left(y_{2}^{\prime}, z_{z}^{\prime}\right) \Psi_{1}^{*}\left(y_{1}^{\prime}, z_{1}^{\prime}\right) d y_{1}^{\prime} d z_{1}^{\prime}\right) \\
& \times \Psi_{1}\left(y_{1}, z_{1}\right) .
\end{aligned}
$$

The coordinates of a point $(y, z)$ can be expressed in the $\left(y_{2}, z_{2}\right)$ and $\left(y_{1}, z_{1}\right)$ coordinate axes as follows:

$$
\begin{aligned}
& y_{2}=y \cos (\theta / 2)-z \sin (\theta / 2), \\
& z_{2}=y \sin (\theta / 2)+z \cos (\theta / 2), \\
& y_{1}=y \cos (\theta / 2)+z \sin (\theta / 2), \\
& z_{1}=-y \sin (\theta / 2)+z \cos (\theta / 2) .
\end{aligned}
$$

The evaluation of $E_{x, 1}^{(1)}\left(y_{1}, z_{1}\right)$ can be simplified by assuming $K\left(z_{2}\right) \approx K\left(z_{1}\right)$ in Eq. (27), which is a good approximation for small angles in view of Eqs. (28b) and (28d). Using this approximation and the relations expressed in Eqs. (23) and (24) in Eq. (27) gives

$$
\begin{aligned}
E_{x, 1}^{(1)}\left(y_{1}, z_{1}\right) & \approx\left(i^{2} \int_{-\infty}^{z_{1}} K\left(z_{1}^{\prime}\right) \kappa\left(z_{1}^{\prime}\right) d z_{1}^{\prime}\right) \Psi_{1}\left(y_{1}, z_{1}\right) \\
& =\left\{\left[i K\left(z_{1}\right)\right]^{2} / 2 !\right\} \Psi_{1}\left(y_{1}, z_{1}\right) .
\end{aligned}
$$

In a similar manner, it is shown that for $n \geqslant 1$

$$
\begin{aligned}
& E_{x, 2}^{(n)}\left(y_{2}, z_{2}\right) \approx\left\{\left[i K\left(z_{2}\right)\right]^{2 n-1} /(2 n-1) !\right\} \Psi_{2}\left(y_{2}, z_{2}\right), \\
& E_{x, 1}^{(n)}\left(y_{1}, z_{1}\right) \approx\left\{\left[i K\left(z_{1}\right)\right]^{2 n} /(2 n) !\right\} \Psi_{1}\left(y_{1}, z_{1}\right) .
\end{aligned}
$$

Equations (4a), (4b), (9), (25a), (30a), and (30b) are used to obtain the total field in the two waveguides as

$$
\begin{aligned}
& E_{x, 2}\left(y_{2}, z_{2}\right)=i \sin \left[K\left(z_{2}\right)\right] \Psi_{2}\left(y_{2}, z_{2}\right), \\
& E_{x, 1}\left(y_{1}, z_{1}\right)=\cos \left[K\left(z_{1}\right)\right] \Psi_{1}\left(y_{1}, z_{1}\right) .
\end{aligned}
$$

At finite values of $z_{2}$ and $z_{1}$ it is not meaningful to speak of the amount of power in individual waveguides, because the two waveguides are decoupled only at $z_{2,1} \rightarrow \pm \infty$. In order to calculate the power in waveguides 2,1 as a consequence of coupling between them, we take the limit of Eqs. (31a), and (31b) as $z_{2,1} \rightarrow+\infty$. Since the eigenfunctions $\Psi_{i}\left(y_{i}, z_{i}\right)$ with $i=1,2$ are normalized to unit power, the power transferred to waveguide 2 is given by

$$
P_{\times}=\sin ^{2}\left(K_{0}\right)
$$

and the unconverted power in waveguide 1 is given by

$$
P==\cos ^{2}\left(K_{0}\right) \text {, }
$$

where

$$
K_{0} \equiv \lim _{z_{2} \rightarrow+\infty} K\left(z_{2}\right)=\lim _{z_{1} \rightarrow+\infty} K\left(z_{1}\right) .
$$

It should be noted that the convention for classifying the TE and TM modes for a slab and a channel waveguide is different. Therefore, the TE (TM) modes of a slab waveguide are physically equivalent to the TM (TE) modes of a channel waveguide. In the remainder of this section the coupling between the identical intersecting waveguides is discussed for two specific choices of the scattering inhomogeneity in the intersection region.

\section{A. Single- $\Delta \boldsymbol{n}$ intersecting waveguides}

The single- $\Delta n$ intersecting waveguides are obtained by taking

$$
\iota(y, z)=-\left(\epsilon_{f}-\epsilon_{s}\right) \equiv-\Delta,
$$

in the intersection region. In such a case the coupling coefficient $K_{0}$ represents the coupling due to the overlap of one of the waveguides with the evanescent tail of the field in the 


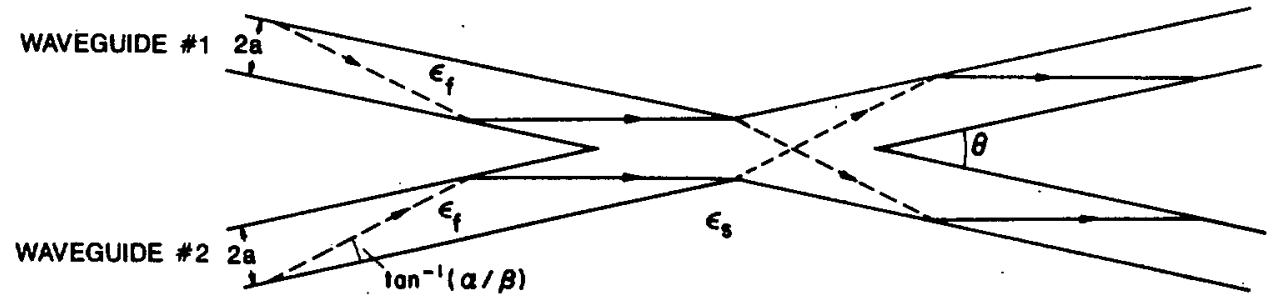

FIG. 3. Rays are drawn in the two waveguides which are intersecting at an angle $\theta$ such that $\theta=2 \tan ^{-1}(\alpha)$ $\beta$ ). It should be noted that the rays are parallel for half of the bounce distance and that no particular point of origin of the rays is implied in either of the two waveguides.

$\theta=2 \tan ^{-1}(a / \beta)$

other and is given by

$$
K_{0}=K_{0}^{e v} \text {, }
$$

where

$$
\begin{aligned}
K_{0}^{\mathrm{ev}}= & \frac{\omega^{2} \mu \Delta}{\beta(a+1 / \gamma) \sqrt{\left(1+\gamma^{2} / \alpha^{2}\right)}\left[\gamma^{2}+\beta^{2} \tan ^{2}(\theta / 2)\right] \sin \theta}\{\gamma \cos [\beta \tan (\theta / 2) a]-\beta \tan (\theta / 2) \sin [\beta \tan (\theta / 2) a]\} \\
& \times\left(\frac{\sin \{[\alpha-\beta \tan (\theta / 2)] a\}}{[\alpha-\beta \tan (\theta / 2)]}+\frac{\sin \{[\alpha+\beta \tan (\theta / 2)] a\}}{[\alpha+\beta \tan (\theta / 2)]}\right) .
\end{aligned}
$$

The values of $\alpha, \beta, \gamma$ are determined by solving the dispersion relation

$$
\gamma=\alpha \tan (\alpha a)
$$

in terms of the normalized parameters ${ }^{8}$

$$
V=\left(4 \pi \sqrt{n_{f}^{2}-n_{s}^{2}} a\right) / \lambda
$$

and

$$
b=\left(N^{2}-n_{s}^{2}\right) /\left(n_{f}^{2}-n_{s}^{2}\right),
$$

where $n_{f}=\sqrt{\epsilon_{f} / \epsilon_{0}}, n_{s}=\sqrt{\epsilon_{s} / \epsilon_{0}}$, and $N$ is the effective index of the slab waveguide. The single-mode waveguides are characterized by $0 \leqslant V \leqslant \pi$, where a larger value of $V$ corresponds to a better confined mode. It is seen from Eqs. (34b) and (35a) that

$$
K_{0}^{\text {ev }}=0
$$

when

$$
\alpha=\beta \tan (\theta / 2),
$$

because at this condition

$$
\begin{aligned}
& \gamma \cos [\beta \tan (\theta / 2) a] \\
& \quad-\beta \tan (\theta / 2) \sin [\beta \tan (\theta / 2) a]=0 .
\end{aligned}
$$

The intersection angle for which Eq. (36b) is satisfied is hereafter referred to as magic angle. It is obvious that there is a range of possible values for the magic depending on the value of $V$. As a general rule, the value of the magic angle decreases with increasing $V$, attaining its minimum value at $V=\pi$ for a given value of $n_{f}$ and $n_{s}$. Since the inclination angle of the rays in the two waveguides is equal to $\tan ^{-1}(\alpha /$ $\beta$ ) with respect to their axes (see Fig. 3), the magic angle of intersection is simply twice the ray angle. In Fig. 3, rays are drawn in the two waveguides which are intersecting at the magic angle. It should be noted that at this angle of intersection the propagation wave vectors or rays are parallel for half of the bounce distance in the two waveguides. Therefore; Eq. (36b) also represents a quasi-phase-matching condition. The effect of the phase-matching condition manifests itself in the expression for $K_{0}^{\text {ev }}$ in terms of two sinc functions [see Eq. (34b)]. Numerical analysis has shown that a typical value of the magic angle is $5^{\circ}$. It is also found that the resonance represented by the sinc functions with respect to the mode confinement parameter $V$ is quite broad and hence, does not yield any new features for evanescent coupling between intersecting waveguides. The value of $K_{0}^{\text {ev }}$ passes over smoothly from positive to negative values including zero as the value of $V$ is increased from 0 to $\pi$ (see Fig. 4). The cusp seen in Fig. 4 arises due to the fact that it is the absolute value of $K_{0}^{\mathrm{ev}}$ that is plotted. Due to the $\propto(\sin \theta)^{-1}$ dependence of $K_{0}^{e v}$, the effective number of coupling lengths in an intersecting waveguide device increases for decreasing values of $\theta$. If

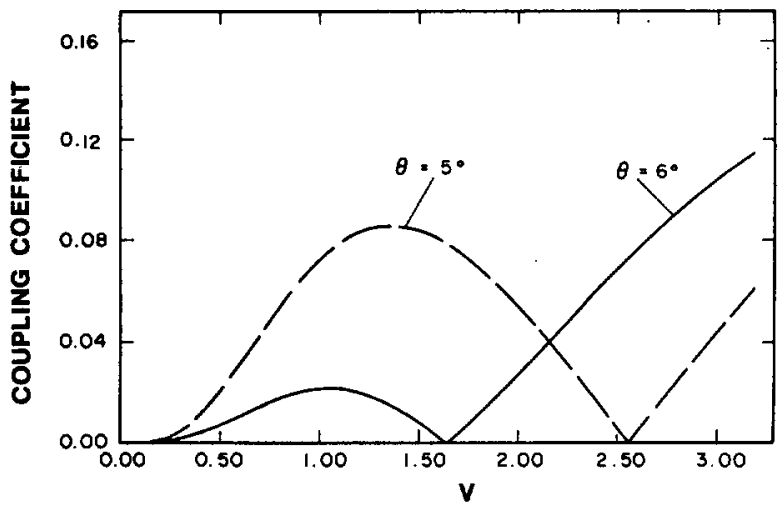

FIG. 4. Evanescent coupling coefficient $\left|K_{0}^{e v}\right|$ as a function of mode confinement parameter $V$ for two slab waveguides intersecting at angles of $5^{\circ}$ and $6^{\circ}$. The waveguide parameters are $a=2 \mu \mathrm{m}, n_{s}=2.300$, and $n_{f}=2.305$. 


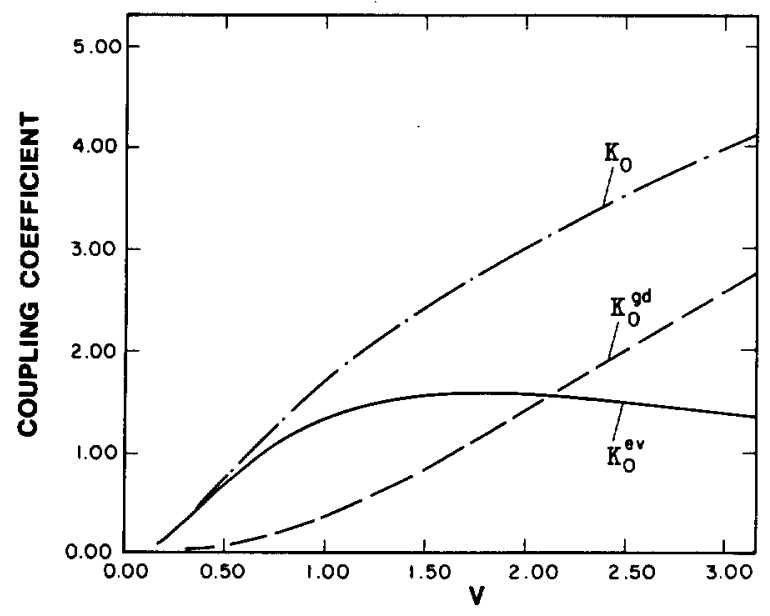

FIG. 5. Evanescent ( $\left.K_{0}^{\mathrm{ev}}\right)$, guided $\left(K_{0}^{\mathrm{gd}}\right)$, and total $\left(K_{0}\right)$, integrated coupling coefficients as a function of $V$ for two slab waveguides intersecting at a small angle $\left(\theta=1^{\circ}\right)$. The strength of coupling coefficients is plotted in terms of the number of coupling lengths. The waveguide parameters are $a=2 \mu \mathrm{m}, n_{s}=2.300$, and $n_{f}=2.304$.

the angle of intersection is less than the magic angle corresponding to $V=\pi$ for a given value of $n_{f}$ and $n_{s}$, no cusp is observed in $K_{0}^{\text {ev }}$ vs $V$. Instead $K_{0}^{\text {ev }}$ will increase, reach a broad maximum, and then decrease with increasing $V$, which is to be expected from the familiar properties (e.g., in a directional coupler) of evanescent coupling (see Fig. 5).

\section{B. Double- $\Delta n$ intersecting waveguides}

The double- $\Delta n$ intersecting waveguides are obtained by taking $\iota(y, z)$ equal to zero in the intersection region. In this case, the coupling coefficient has an additional component due to the overlap of one of the waveguides with the guided fields in the other and is given by

$$
K_{0}=K_{0}^{\mathrm{ev}}+K_{0}^{\mathrm{gd}} \text {, }
$$

where the expression for $K_{0}^{\text {ev }}$ has already been obtained in Eq. (34b). The coupling coefficient $K_{0}^{\text {gd }}$ is given by

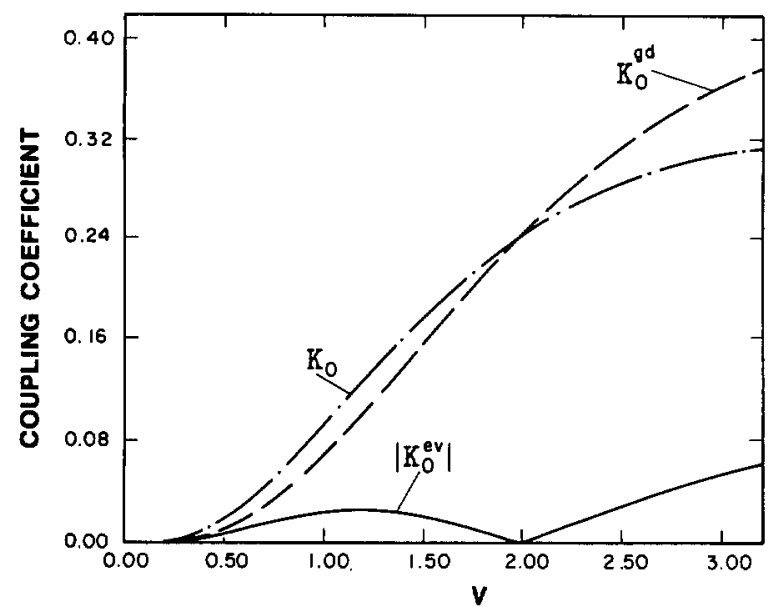

FIG. 6. Evanescent $\left(K_{0}^{\mathrm{cv}}\right)$, guided $\left(K_{0}^{\mathrm{gd}}\right)$, and total $\left(K_{0}\right)$, integrated coupling coefficients as a function of $V$ for two slab waveguides intersecting at a large angle $\left(\theta=5^{\circ}\right)$. The strength of coupling coefficients is plotted in terms of the number of coupling lengths. The waveguide parameters are $a=2 \mu \mathrm{m}, n_{s}=2.300$, and $n_{f}=2.304$.

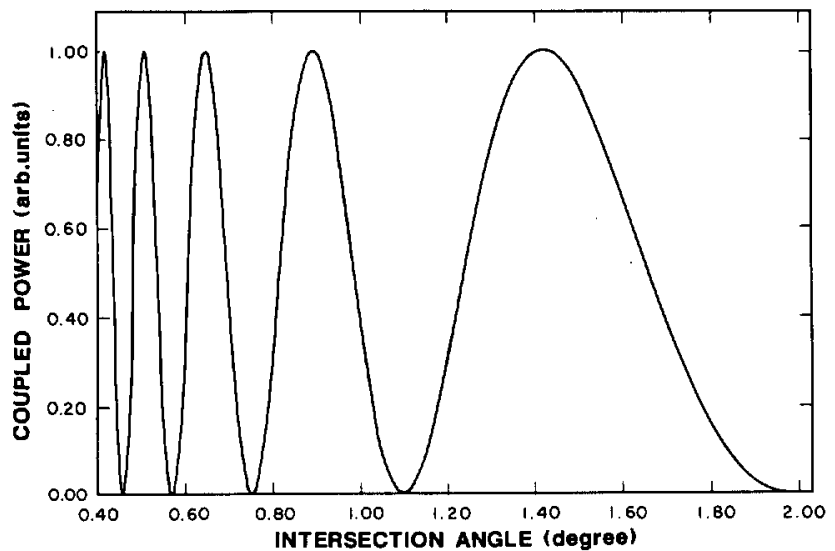

FIG. 7. The coupled power $\left[P_{\times}=\sin ^{2}\left(K_{0}\right)\right]$ from waveguide 1 to 2 as a function of the intersection angle for the double- $\Delta n$ intersecting waveguides. The parameters $a=2 \mu \mathrm{m}, n_{s}=2.300$, and $n_{f}=2.305$ are selected so as to obtain single-mode waveguides at $\lambda=1.3 \mu \mathrm{m}$.

$$
\begin{aligned}
K_{0}^{\mathrm{gd}} & \frac{\omega^{2} \mu \Delta}{2 \beta[a+(1 / \gamma)] \sin \theta}\left(\frac{\sin \{[\alpha-\beta \tan (\theta / 2)] a\}}{[\alpha-\beta \tan (\theta / 2)]}\right. \\
& \left.+\frac{\sin \{[\alpha+\beta \tan (\theta / 2)] a\}}{[\alpha+\beta \tan (\theta / 2)]}\right)^{2}
\end{aligned}
$$

The dependence of $K_{0}^{\mathrm{gd}}$ as a function of $V$ is shown in Figs. 5 and 6 for a small and a large angle of intersection, respectively. The most important aspect of this coupling is the monotonic increase in the value of $K_{0}^{\text {gd }}$ with the mode confinement parameter $V$. This behavior is in contrast with that of the evanescent coupling in intersecting waveguides. It turns out that the guided wave coupling is stronger than evanescent coupling in the case of intersecting waveguides under consideration, so that the total coupling coefficient $K_{0}$ has the same dependence on $V$ as of $K_{0}^{\text {gd }}$ (see Figs. 5 and 6).

We conclude this section by describing the coupling between the two intersecting waveguides as a function of their angle of intersection. There are two distinct regimes of operation of these devices depending on the magnitude of the intersection angle $\theta$. In the limit of smaller values of $\theta$ $\left(\Sigma 1^{\circ}\right)$, the interaction between the two waveguides corre-

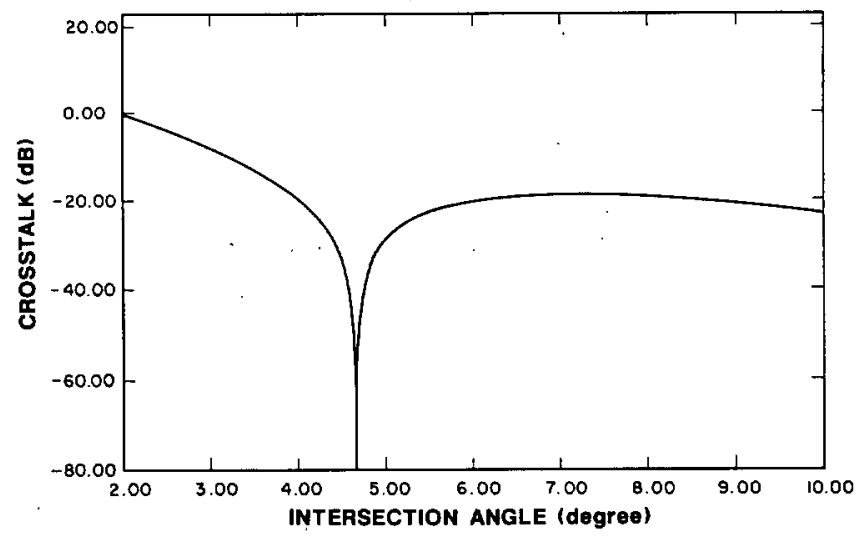

FIG. 8. The crosstalk $\left(P_{\times} \approx 10 \log \left(\left|K_{0}\right|^{2}\right)\right)$ between waveguide 1 and 2 as a function of the intersection angle for the single- $\Delta n$ intersecting waveguides. The parameters $a=2 \mu \mathrm{m}, n_{s}=2.300$, and $n_{f}=2.305$ are selected so as to obtain single-mode waveguides at $\lambda=1.3 \mu \mathrm{m}$. 
sponds to several coupling lengths. A rapid variation in the number of coupling lengths with the intersection angle is responsible for the oscillatory nature of the coupling shown in Fig. 7. Such results are in agreement with the experimental data obtained with double- $\Delta n$ intersecting waveguides. ${ }^{9}$ In the limit of larger values of $\theta\left(\gtrsim 3^{\circ}\right)$, the crosstalk can be written as

$$
P_{\times} \approx\left|K_{0}\right|^{2}
$$

Such an approximation is quite good for single- $\Delta n$ intersecting waveguides, because in this limit $k_{\theta} \ll 1$. Thus, the contribution of higher-order scattering interactions is negligible [see Eqs. (30a) and (30b) ]. Moreover, for larger values of $\theta$ the phase-matching condition can be satisfied for singlemode waveguides. Thus, the evanescent coupling does vanish in this regime of operation for a particular value of $V$. It is seen from Fig. 8 that crosstalk between the two waveguides vanishes at the magic angle of intersection for single- $\Delta n$ intersecting waveguides. Such results are in good quantitative agreement with the experiments. ${ }^{10}$

\section{CONCLUDING REMARKS}

We have developed a multiple scattering technique to analyze the coupling between two parallel and intersecting dielectric waveguides. The solutions obtained for parallel waveguides by this technique are in agreement with those derived from the coupled mode theory. For intersecting waveguides, the analysis is restricted to identical waveguides. It is noteworthy that the multiple scattering interaction analysis is essentially an analytical technique in the limit of a large strength of interaction between the two waveguides. This fact is of crucial importance because in such a case the infinite series obtained in this analysis cannot be summed up by numerical methods.

The previous theory for the analysis of intersecting waveguides which invokes the concept of mode interference makes two key assumptions. First, the existence of local modes of the composite structure is postulated and second, the phase shift between them is interpreted as the coupling coefficient. The first of these assumptions is quite good at small angles of intersection. However, the second assumption which tacitly assumes a weak coupling between the two intersecting waveguides is invalid regardless of the size of intersection angle. In addition, there is no sensible way to model the interaction between the two waveguides in the intersection region. In general both qualitative and quantitative discrepancies remain between this theory and the experiments.

Some aspects of the coupling between two intersecting waveguides have been treated by the beam propagation method. ${ }^{6,11}$ In this method, a single propagation direction is identified, the rapidly varying phase in that direction is separated out and the slowly evolving transverse field distribution is evaluated numerically under the assumption that the changes in the geometry and the material parameters in the propagation direction are sufficiently small so as not to introduce any reflected waves. In the method developed in this paper, the two propagation directions corresponding to the axes of the two intersecting waveguides are introduced at the outset and the reflected and radiated waves can be taken into account if needed. Thus, the multiple scattering interaction analysis of the intersecting waveguides is general.

The experimental measurements ${ }^{10}$ on the crosstalk and loss characteristics of intersecting waveguides do not agree with the results obtained using a two-dimensional beam propagation method in conjunction with the concept of effective index. ${ }^{6}$ It has been suggested that the validity of the assumptions inherent in such an analysis is questionable in the context of intersecting waveguides. Therefore, a numerical analysis employing a three-dimensional beam propagation method that eliminates the need to use the approximation of effective index has been carried out. ${ }^{11}$ However, this improved numerical analysis still yields results which do not agree completely with the experiments. The crosstalk measurements on single- $\Delta n$ intersecting waveguides as a function of intersection angle are in good agreement with the analysis presented here as pointed out previously. In addition, an extension of the multiple scattering analysis to include the coupling between guided and radiation modes of the individual waveguides is carried out and will be reported elsewhere. It is found that such analysis agrees both qualitatively and quantitatively with the experimental data on insertion loss. ${ }^{10}$

The unique features of coupling between intersecting waveguides described in this paper are of great practical signifiance in terms of device applications. As the complexity of optical integrated circuits increases, it becomes important to be able to cross one waveguide over the other without any cross talk. In such applications all that needs to be done is to let the different waveguides intersect each other at the magic angle. The increase in the total coupling coefficient with mode confinement for two intersecting waveguides is of added importance since it leads to a requirement of smaller asymptotic separation between the two waveguides. Such a property is invaluable, since it can be used to design still smaller devices with intersecting waveguides as compared to directional couplers.

\section{ACKNOWLEDGMENTS}

This research was supported by the National Science Foundation under Grants Nos. ECS-8504513 and ECS8508024.

${ }^{1}$ C. S. Tsai, B. Kim, and F. R. E. Akkari, IEEE J. Quantum Electron. QE14, 513 (1978).

${ }^{2}$ H. Nakajima, T. Horimatsu, M. Sieno, and I. Sawaki, IEEE Trans. Microwave Theory Tech. MTT-30, 617 (1982).

${ }^{3}$ A. Neyer, Electron. Lett. 19, 553 (1983).

${ }^{4}$ A. Neyer, W. Mevenkamp, and B. Kretzschmann, in Technical Digest of the Topical Meeting on Integrated and Guided-Wave Optics 1986, Atlanta, Georgia (Optical Society of America, Washington, DC, 1986), pp.4 and 5.

${ }^{5}$ L. McCaughan, Proc. Soc. Photo-Opt. Instrum. Eng. 517, 246 (1984).

${ }^{6}$ A. Neyer, W. Mevenkamp, L. Thylen, and B. Lagerström, J. Lightwave Technol. LT-3, 635 (1985)

${ }^{7}$ A. Yariv and P. Yeh, Optical Waves in Crystals (Wiley, New York, 1984), Chap. 6, pp. 186 and 187.

${ }^{8}$ H. Kogelnik and V. Ramaswamy, Appl. Opt. 13, 1857 (1974).

${ }^{9}$ E. E. Bergmann, L. McCaughan, and J. E. Watson, Appl. Opt. 23, 3000 (1984).

${ }^{10} \mathrm{G}$. A. Bogert, Electron. Lett. 23, 72 (1987).

"M. D. Feit and J. A. Fleck, IEEE J. Quantum Electron. QE-21, 1799 (1985). 